MKG-Chirurg 2011 · 4:78-78

DOI 10.1007/s12285-010-0209-8

Online publiziert: 10. April 2011

(c) Springer-Verlag 2011

\author{
J.T. Lambrecht \\ Klinik für Zahnärztliche Chirurgie, - Radiologie, Mund- und Kieferheilkunde, \\ Universität Basel
}

\title{
Dentoalveoläres Trauma
}

Dentoalveoläre Traumata ereignen sich häufig. In der Bevölkerung existieren eher rudimentäre Kenntnisse über das korrekte Verhalten am Unfallort bzw. über die suffiziente Erstversorgung in der zahnärztlich-chirurgischen oder MKG-chirurgischen Praxis. Das Ziel ist, unfallgeschädigte Zähne lebenslang zu erhalten. Dies gelingt nicht immer, da es sich oft um komplexe Verletzungen handelt, die mehrere Gewebe betreffen: Zahnhartsubstanzen, Pulpa, Parodont, Knochen und umgebende Weichgewebe. Diese müssen unterschiedlich behandelt werden. Fehlentscheidungen am Unfalltag bezüglich der Behandlungen von Verletzungen der Pulpa oder des Parodonts resultieren häufig in frühzeitigem Zahnverlust. Die so entstandene Lücke führt während des weiteren Kieferwachstums zu Unsicherheiten bezüglich des lokalen Gewebeerhalts über kieferorthopädische Planungen bis hin zu Diskussionen über mögliche autologe Transplantationen. Die Therapiekonzepte nach Zahntrauma haben sich in den letzten Jahren teilweise erheblich verändert und werden dies auch weiter tun. Der Beitrag von A. Filippi dokumentiert den augenblicklichen Stand des Wissens. An der Universität Basel wurde das Zahnunfallzentrum etabliert. Dies ist ein Gemeinschaftsprojekt zwischen der Klinik für Zahnärztliche Chirurgie, Radiologie, Mund- und Kieferkrankheiten, der Klinik für Parodontologie, Endontologie und Kariologie sowie der Schulzahnklinik Basel-Stadt. Nicht nur primäre dentoalveoläre Zahntraumata, die von den niedergelassenen Kollegen überwiesen werden, kommen zur Behandlung, sondern auch heranwachsende Kinder und Jugendliche sowie Erwachsene, die mit Spätfolgen zu tun haben. Diese segensreiche Einrichtung zum Wohl der meist jungen Patien- ten, die auf diese Weise auch schwer verletzte Zähne lebenslang behalten können, ist durchaus zur Nachahmung empfohlen.

\section{(7) Ziel der Versorgung ist die lebenslange Erhaltung unfallgeschädigter Zähne}

Eine mögliche Therapieform des dentoalveolären Traumas ist die autogene Zahntransplantation, hier werden von $D$. Nolte et al. neue Perspektiven aufgezeigt. Zahntransplantationen sind grundsätzlich in jedem Alter möglich; sinnvoll und vorhersagbar erfolgreich sind sie aber nur innerhalb desselben Individuums sowie bei Kinder und Jugendlichen. Heute existieren gute wissenschaftliche Informationen über die biologischen Abläufe nach Zahntransplantation. Auch die jeweiligen Risikofaktoren für den Misserfolg sind bekannt und bedingt beeinflussbar. Die Erfolgsraten nach Zahntransplantation müssen sich hinter denen von Implantaten nicht mehr verstecken. $\mathrm{Zu}$ groß waren der Wissenszugewinn und der Qualitätsschub in den letzten Jahren.

Neben den Unfällen im Alltag kommt es auch an der Schnittstelle zwischen Human- und Zahnmedizin während Intubationsnarkosen häufiger zu Zahnverletzungen. Im ihrem Beitrag berichten H. Dep$p e$ et al. über die Häufigkeit und Ätiologie der periintubationsbedingten Zahnschäden. Zur Vermeidung periintubationsbedingter Frontzahntraumen sollte eine sorgfältige Gebissevaluation erfolgen. Neben der zahnärztlichen Versorgung ist auch eine entsprechende Dokumentation zu berücksichtigen.

Die dentoalveoläre Traumatologie ist ein Teilgebiet der zahnärztlichen Chirurgie, die in den letzten 30 Jahren auf eine erstaunliche Entwicklung zurückblicken kann. Die Änderung der Ausstattung sowohl im diagnostischen Bereich (digitales Röntgen, digitale 3-D-Volumentomographie) als auch im apparativen Bereich $\left(\mathrm{CO}_{2}\right.$-Laser, Piezochirurgie, Operationsmikroskop, starre und flexible Endoskopie) und nicht zuletzt die Entwicklungen im Bereich der Implantologie und der Biomaterialien sind die Grundlage für einen völligen Wandel im zahnärztlich-chirurgischen Alltag, die auch Auswirkungen auf die dentoalveoläre Traumatologie haben. Es ist der Schriftleitung des Organs der Deutschen Gesellschaft für Mund-, Kiefer- und Gesichtschirurgie Der MKG-Chirurg zu danken, dass sie dies zu einem ihrer Schwerpunktthemen gemacht hat.

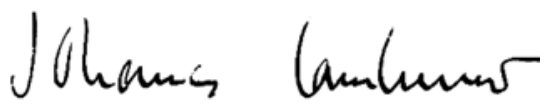

Prof. Dr. Dr. J.T. Lambrecht

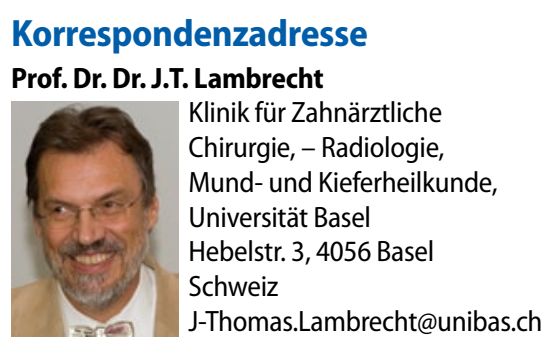

